\title{
Esiste oggi un ruolo per l'emodialisi domiciliare e che cos'è oggi l'emodialisi domiciliare?
}

\author{
Giorgina Barbara Piccoli, Martina Ferraresi, Federica Neve Vigotti, Gerardo Di Giorgio
}

S.S. Nefrologia e Dialisi, A.S.O.U. San Luigi Gonzaga, Orbassano; Dipartimento di Scienze Cliniche e Biologiche, Università degli Studi di Torino, Torino

\begin{abstract}
IS THERE A ROLE FOR HOME DIALYSIS AND WHAT IS HOME DIALYSIS TODAY?
Abstract. The global economic crisis, the pressure toward de-hospitalization of chronic patients, and the innovations in the treatment of chronic uremia are the basis of the increased interest in "non-conventional or intensive" dialysis treatments, often necessarily performed at home. A first point highlighted in this narrative review is the overcoming of the historical antagonism between home hemodialysis (HHD) and peritoneal dialysis (PD), by the motto "home dialysis first". In fact, justifying the decreased use of an HHD technique with the competition with another technique, PD, is as simplistic as considering that the ideal HHD patients are those who dropped out from PD. On the contrary, PD and HHD may even potentiate one another in settings requiring specific care.

In this review we analyzed the issue of home dialysis also according to the four principles of the so-called principlism bioethics: beneficium, non maleficium, justice, and autonomy.

If the benefit is not easy to prove, mainly because of the peculiar selection of the patients, the non maleficium is evident: in all the studies analyzed home hemodialysis (standard, daily, or intensive) led to results that were never significantly lower than conventional treatments, and were actually superior in most of the cases. The principle of justice, considered in a reductive way as "distributive" economical justice, was analyzed by evaluating the cost of treatment. Once more the issue is complex; in brief, direct costs were divided in costs of disposables and machines, and cost of medical and nursing staff. The economic benefit of reducing the workforce is obvious; however, a home treatment needs a "critical mass" of patients to be favorable from the economic point of view. Furthermore, the "indirect" costs (e.g., the hospital "structure") are difficult to quantify. In any case, in no study the cost of HHD was higher than that of in-center HD. The fourth principle is patient's autonomy: there is however no choice without offer. Hence, physicians should be able to offer HHD to all patients without contraindications, by creating accessible reference centers where patients can be counseled by doctors, nurses, and patients with specific experience.
\end{abstract}

Key words: Home dialysis, Hemodialysis, Peritoneal dialysis, Principles ethic, Intensive dialysis

Conflict of interest: None.

Financial support: None.

Accettato: 14 Aprile 2014

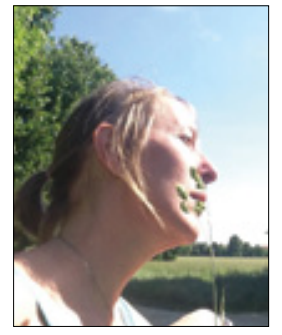

Giorgina Barbara Piccoli

A oltre 50 anni dalle prime emodialisi croniche, la terapia sostitutiva dell'uremia cronica sta conoscendo un'interessante rivisitazione critica in tutto il mondo, sulla scorta di numerosi fattori, clinici e sociali: la crisi economica globale, che porta a una revisione dei costi e delle indicazioni dei trattamenti più costosi e diffusi, come la dialisi, la tendenza alla massima deospedalizzazione dei pazienti cronici, con la strutturazione di percorsi a differente intensità di cura, e le novità tecnologiche e di ricerca nel campo del trattamento dell'uremia cronica.

Sono centrali in questo processo il rinnovato interesse per l'emodialisi domiciliare (HHD) e, legato a questo, l'interesse per trattamenti variamente definiti come "non convenzionali o intensivi", che non solo offrono una serie di spunti interessanti per quanto riguarda l'organizzazione del trattamento, ma, attraverso i risultati delle dialisi "notturne lunghe", dimostrano anche fino a che punto può essere spinta la sostituzione della funzione renale attraverso l'emodialisi (1-3).

\section{Una breve revisione bibliografica}

A metà Marzo 2014, interrogando Medline con una strategia di ricerca super-semplificata ("home hemodialysis" come termine di ricerca libera), si ritrovano 1898 titoli, di cui un po' più di un quarto negli ultimi 10 anni, a riprova di un interesse limitato, ma non spento sull'argomento (538 articoli, 
359 nell'ultimo quinquennio); il numero aumenta sensibilmente se si aggiungono i termini relativi all'emodialisi notturna, trattamento per definizione domiciliare (658 articoli nell'ultima decade). Non sono numeri irrilevanti, ma la differenza nei confronti della dialisi peritoneale, l'altro "grande" trattamento domiciliare, è notevole: la semplice ricerca "Peritoneal dialysis" recupera ben 8506 titoli di articoli pubblicati negli ultimi 10 anni.

La tipologia dei lavori recenti disponibili è molto varia, da lavori di tipo storico ad analisi biochimiche, con ampio spazio agli aspetti logistici ed economici del trattamento (4-15). La sovrapposizione, nell'ultima decade, con le dialisi intensive è così elevata da suggerire di prendere in considerazione i due argomenti come un tutt'uno (16-30), sottolineando già da subito come il rinnovato interesse per la dialisi domiciliare sia almeno in parte legato alla disponibilità di nuovi approcci dialitici.

In questa revisione narrativa della letteratura, che ha lo scopo di suscitare interesse e discussione, più che l'ambizione di essere esaustiva, prenderemo in considerazione alcuni aspetti del problema: il rapporto con la dialisi peritoneale, il rapporto con le dialisi intensive, la scomposizione "bioetica" del problema della disponibilità dell'emodialisi domiciliare e i principali modelli di riferimento, che hanno supportato la ripresa dell'emodialisi domiciliare nel mondo. L'ampia bibliografia fornita ha lo scopo di fornire una "chiave di ingresso" dettagliata per chi desideri avviare questo affascinante percorso clinico e assistenziale.

\section{Emodialisi domiciliare e dialisi peritoneale: alleate $o$ antagoniste?}

Il rischio che la più complessa emodialisi domiciliare possa soppiantare la dialisi peritoneale (PD) è riferito spesso e il timore è segnalato particolarmente dai Centri che non offrono entrambe le opzioni; in realtà, non vi sono dati in letteratura a favore di un reale antagonismo tra le due metodiche, al momento attuale. Al contrario, in diversi ambiti, i trattamenti dialitici domiciliari vengono seguiti assieme, da un team medico-infermieristico dedicato. Questa scelta, che ha avuto nel nuovo millennio un grande sostenitore nel professor Oreopoulos, propugnatore dell'antico motto "home dialysis first", porta a un superamento di un antagonismo storico tra emodialisi domiciliare e PD (31). L'avversione da parte di esperti di dialisi peritoneale per l'emodialisi domiciliare, vista come potenziale competitore, in effetti, rappresenta una sorta di paradosso storico, che interpreta la diminuzione dell'emodialisi domiciliare come legata alla disponibilità di una dialisi peritoneale sempre più semplice e sicura e che teme, quindi, un "rovesciamento delle parti" al momento di un rilancio di un'emodialisi domiciliare migliorata e semplificata. Questa interpretazione riduzionista di un sistema complesso dimentica che, contemporaneamente all'incremento della PD e al decremento dell'HHD, si assisteva allo sviluppo, nella maggior parte d'Europa, di reti di piccoli Centri emodialitici decentrati, cosiddetti "satellite" o "ad assistenza limitata" e alla maggiore disponibilità di trapianto renale (32). Ascrivere il successo di una metodica domiciliare alla competizione con l'altra è ridut- tivo quanto il considerare che i pazienti ideali per la PD siano principalmente $i$ soggetti che hanno dovuto interrompere la dialisi peritoneale: anche in questo caso, la letteratura non avvalora una scala di scelte dalla PD all'emodialisi domiciliare alla dialisi ospedaliera, ma, piuttosto, una partenza associata e in parallelo delle due metodiche, con la possibilità di accesso a entrambe in qualsiasi punto del sistema (33-36).

In un recente lavoro di Toronto, da parte proprio della scuola di Oreopoulos, ben nota per gli eccellenti risultati ottenuti con entrambi i trattamenti, sia la sopravvivenza del paziente sia la durata del trattamento domiciliare sono sovrapponibili tra chi ha iniziato l'emodialisi domiciliare con o senza una precedente "esposizione" alla peritoneale (37). Tra gli oltre 200 pazienti seguiti dal gruppo, solo il $17 \%$ proveniva dalla dialisi peritoneale: una percentuale rilevante, ma che acquista un significato solo in un sistema che offre entrambi i tipi di dialisi domiciliari, e che raggiunge la massa critica di pazienti proponendola sistematicamente a tutti i livelli. In effetti, l'assenza di competizione tra HHD e PD è ribadita da tutti gli esperti di dialisi domiciliare nel nuovo millennio, sia dall'ottica più dell'emodialisi sia da quella della PD stessa, come coloritamente espresso in un recente editoriale di Burkard, su Hemodialysis International intitolato (38): il ruolo della PD nell'era della resurrezione dell'HHD: "non c'è da avere paura che la PD sia cannibalizzata dall'HHD'. L'articolo porta una serie di esempi di felice concomitanza dei due programmi, che paiono convivere, al contrario, pacificamente, con una minima sovrapposizione di indicazioni, se si considera che, globalmente, meno del $10 \%$ dei pazienti passa dalla PD all'HHD, una percentuale inferiore certamente a quanto atteso in caso di una sovrapposizione delle indicazioni.

Il modello home dialysis first pare funzionare dovunque, dalla Finlandia $(39,40)$, che ha rilanciato recentemente un programma, ora attivo, agli Usa e al Canada: anche se ancora distante dall'elevatissima prevalenza di pazienti a domicilio in Australia e in Nuova Zelanda, l'attenzione all'emodialisi domiciliare tende a convivere addirittura con un aumento della peritoneale e non viceversa. È centrale, nello sviluppo di strategie di implementazione, il problema organizzativo e dei costi, che verrà discusso nel prossimo paragrafo (41-60).

Nel complesso, la prevalenza dell'emodialisi domiciliare non supera il $5 \%$ dei casi nel mondo, pur con alcune punte superiori in Australia e in Nuova Zelanda; le implicazioni economiche di un programma di emodialisi domiciliare sono, però, enormi, anche con numeri così piccoli; questi aspetti, uniti alle necessità organizzative, saranno discussi nei prossimi paragrafi.

\section{Dialisi quotidiane, intensive, notturne: il valore aggiunto dell'emodialisi domiciliare}

Non ci sono dubbi: le dialisi quotidiane, notturne, flessibili e intensive rappresentano una nuova frontiera in emodialisi (16-18, 31, 41, 42, 44-46, 48, 49, 52, 56, 57). Così come la dialisi domiciliare, arricchiscono e, al contempo, mettono in crisi un sistema che pareva ormai ben assestato su una prevedibile routine. Così come per l'emodialisi domiciliare, questi trattamenti incontrano accesi sostenitori e acrimoniosi detrat- 
tori, richiedono una struttura organizzativa ad hoc e sono disponibili solo in una limitata minoranza di sedi, ed è proprio la disponibilità di nicchia che rischia di estremizzare le posizioni di difesa e di critica e di riproporre la famosa parabola della volpe e dell'uva. Anche l'emodialisi quotidiana, nelle sue varie forme e con i differenti sinonimi, è una nicchia: su Medline compaiono, sempre al Marzo 2014, 690 titoli, combinando i termini di ricerca "libera" daily dialysis, daily hemodialysis, more frequent dialysis, intensive dialysis e nocturnal dialysis, 387 negli ultimi 10 anni e 184 negli ultimi cinque. Ancora una volta, si tratta di lavori di tutti i tipi, con una tendenza, però, a una numerosità e a una qualità sempre maggiori, a partire dai primissimi, pionieristici studi, molti dei quali di scuola italiana (61-71). La prima ampia revisione sistematica, pubblicata nel 2006 su Clinical $J A S N$, raccoglie meno di 300 pazienti, in ben 14 sedi, a testimonianza di una "penetranza" molto limitata della metodica (72). Circa metà dei pazienti era in emodialisi domiciliare. Il lavoro sottolinea soprattutto i limiti della bassa numerosità e del breve follow-up dei lavori disponibili; pur con questi limiti, i risultati sono concordemente positivi e si auspica che possano essere confermati da un grande studio randomizzato (72).

Lo studio randomizzato giunge quattro anni dopo e viene pubblicato con la dovuta enfasi sul New England Journal of Medicine $(73,74)$. L'elegante studio randomizzato, di dialisi quotidiana in un Centro dialisi, raduna circa 400 pazienti; $\mathrm{i}$ risultati sono favorevoli per un outcome combinato di mortalità e ipertrofia ventricolare, ma pongono un warning per gli accessi vascolari. I sostenitori della dialisi quotidiana gioiscono, ma i risultati non convincono l'opinione pubblica e $\mathrm{i}$ cosiddetti "health care providers": ci vuole altro per cambiare rotta. Tra i problemi metodologici, che portano a considerare i dati promettenti al massimo un successo parziale, si trova la difficoltà dell'arruolamento, limite, peraltro, comune a tutti gli studi randomizzati controllati che interferiscono pesantemente con la qualità della vita: chi di noi o dei nostri pazienti accetterebbe a cuor leggero di essere "tirato a sorte" per eseguire 3 dialisi convenzionali o 6 dialisi brevi alla settimana? In effetti, di oltre 6000 pazienti valutati, circa 3500 potenzialmente arruolabili, solo un $12 \%$ accetta di partecipare allo studio, una proporzione così piccola da fare dubitare sulla reale informatività del campione $(73,74)$.

Analoghi problemi limitano la generalizzabilità dei risultati, peraltro positivi, ma solo per outcome surrogati, del "frequent nocturnal home hemodialysis trial", che raccoglie 45 pazienti per "braccio" (18). La difficoltà di randomizzazione induce a riflettere sul miglior metodo di analisi dei risultati: è discusso se sia da preferire il disegno del trial, che forza la realtà in un disegno semplificato, per analizzarla meglio, con il rischio di limitare la popolazione a colore che accettano di essere scelti come facenti parte di un gruppo, e di non scegliere in quale braccio stare, o uno studio osservazionale che, al contrario, valorizza la complessità dell'esperienza e che cerca di trarre spiegazioni universali dai casi singoli del mondo reale.

In effetti, in linea con un crescente interesse per studi osservazionali in questo campo, escono, nel 2012, due ampi studi, uno sulla qualità della vita in emodialisi "quotidiana breve" domiciliare, che riporta risultati nettamente favorevoli sui circa 300 pazienti intervistati (75), e un altro, uno studio di Registro, che riporta un vantaggio per outcome "robusti", tra cui la sopravvivenza del paziente, mettendo in campo quasi 2000 casi osservati, che riprova che, con fatica, alti e bassi e discussioni, almeno negli USA, l'emodialisi sta ritornando a casa (76).

In sintesi, alla domanda se la dialisi quotidiana possa rappresentare un valore aggiunto per la ripresa della dialisi domiciliare, si può oggi rispondere in maniera affermativa: i risultati della daily dialysis sono in tutti gli studi uguali o migliori di quelli della dialisi "convenzionale"; in questo ambito, gli studi osservazionali, pur con i limiti della sensibilità al contesto, forniscono risultati migliori rispetto agli RCT, il cui valore è, in generale, limitato da un arruolamento molto scarso.

È possibile che solo un radicale cambiamento di ottica, con una maggiore disponibilità di entrambi i trattamenti (domiciliare e dialisi quotidiana), permetta di uscire dall'impasse dell'attesa dello studio perfetto, implementando l'offerta terapeutica ai pazienti.

\section{Dalla matematica all'etica: il problema dell'offerta della dialisi domiciliare}

La cosiddetta etica dei principi, che scompone i problemi decisionali complessi in quattro grandi principi (beneficio, non maleficio, giustizia e autonomia), si può ben adattare alla domanda se valga o meno la pena di offrire oggi l'emodialisi domiciliare in Italia (77-80).

Il criterio del beneficio non è certo: sebbene i risultati siano complessivamente favorevoli, le prove di un beneficio incontestabile, matematicamente solido e indubitabilmente dimostrato non sono disponibili. Ma quando mai la scelta della dialisi si è basata solo sui vantaggi dimostrati? Un esempio per tutti riguarda la dialisi peritoneale: se ci fosse stato bisogno di una dimostrazione di superiorità, mai peraltro raggiunta, per implementare il trattamento, staremmo ancora ad attendere un nuovo Canusa study, senza permettere a quel 5-30\% di pazienti trattati con successo con la PD di avere accesso a questa opzione $(81,82)$.

In effetti, si addice di più alla scelta di un trattamento un criterio di "non nocere": il non maleficio, che corrisponde al disegno di studio di non inferiorità. In questo senso, supportano fortemente l'implementazione sia della dialisi domiciliare sia della dialisi quotidiana o intensiva i risultati d'abitudine almeno pari, e mai significativamente inferiori, registrati con qualsiasi disegno di studio e in qualsiasi sede.

Il principio della giustizia, tanto più in questo contesto di risorse limitate, è frequentemente considerato come quello di vantaggio o, almeno, di non competizione tra le risorse. Il modello di dialisi quotidiana è sicuramente più costoso, per quanto riguarda la necessità di materiali dialitici; liquidarlo come "troppo costoso" sarebbe però probabilmente ingiusto. Rimandando i dettagli ai modelli organizzativi, il problema dei costi è di estrema complessità e il loro computo dipende strettamente dal contesto, oltre che dal tipo di "item" considerati. Il costo della dialisi riconosce, infatti, tre componenti principali: la prima è relativamente simile, almeno in Europa: si tratta del costo dei "disposable" e della macchina per diali- 
si; sebbene anche la macchina per la dialisi sia acquisibile in maniere differenti e, nel suo costo, conti molto anche il tipo di assistenza tecnica, questa quota di "costi diretti" ammonta in media a 30-50 Euro per dialisi ed è, in fondo, oggi la voce di spesa che più si guarda, quando si parla di riduzione dei costi, ma, di fatto, è la spesa minore. La seconda componente è il costo "umano" del personale medico e infermieristico. Si tratta di una spesa estremamente variabile, che dipende dalle dimensioni e dall'organizzazione del sistema dialitico, nonché dagli stipendi delle singole figure professionali, che variano di un $200-300 \%$ in Europa. Un vantaggio economico in questo senso è intuibile, ma va anche ricordato che un sistema domiciliare necessita di una massa critica per essere favorevole dal punto di vista economico e, conseguentemente, occorrono investimenti anche minimi dedicati all'avvio di un sistema, prima che questo diventi economicamente favorevole. Il fatto che l'emodialisi domiciliare rappresenti una nicchia di trattamento, con un potenziale che viene, in genere, definito tra l'1 e il 5\% dei pazienti, porta, come discusso nei prossimi paragrafi, a stabilire unità di training che seguono aree vaste e che necessitano di un'organizzazione adattata alla realtà locale $(43,45-50)$.

Il terzo item è ancora più complesso e riguarda $\mathrm{i}$ cosiddetti "costi indiretti", vale a dire i costi della struttura ospedaliera. A conoscenza di chi scrive, mancano in Italia (e non solo) dati precisi; d'abitudine viene calcolato un forfait del $20 \%$ rispetto alle spese precedenti, ma è opinione di molti esperti che questo capitolo di spesa possa giungere, in particolare dove il sistema sanitario sia particolarmente farraginoso e burocratizzato, a raddoppiare i costi vivi di qualsiasi trattamento. Poiché i vantaggi dell'emodialisi domiciliare sono soprattutto a questo livello, è ovvio che si tratta di un "non detto" cruciale, spesso non dichiarato non solo per la difficoltà di definire $\mathrm{i}$ costi reali, ma anche per ovvie ragioni politiche, che potrebbero portare tra l'altro a definire un vantaggio per le strutture private, non privo di rischi per la qualità dell'assistenza.

Dalla Tabella, che riporta $\mathrm{i}$ dati già calcolati per la Regione Piemonte e sostanzialmente confermati, anche se con un differente approccio, nella nostra Unità, si può desumere un vantaggio indicativo di 20.000 Euro all'anno rispetto a un trattamento ospedaliero, vantaggio che è certamente sottostimato, tenendo conto del fatto che i costi indiretti sono stati calcolati conservativamente come pari al $20 \%$. In questo senso, giungere a un $2 \%$ di pazienti in emodialisi domiciliare nella nostra regione (circa 3000 pazienti in dialisi, attualmente 20-25 in emodialisi domiciliare) porterebbe a un risparmio di almeno 1.200.000 Euro all'anno (Tab. I).

Infine, un'opinione personale: non è corretto paragonare la bicarbonato dialisi quotidiana alla bicarbonato dialisi trisettimanale: la dialisi quotidiana è una scelta che aumenta sia l'efficienza che la tolleranza. Pertanto, almeno nei Paesi come l'Italia dove vi è un ampio sviluppo dell'emodiafiltrazione, è con questo trattamento che il confronto andrebbe eseguito. Le differenze pesano in particolare per i "disposable": dal momento che, in linea generale, il "kit dialitico" per un'emodiafiltrazione costa il doppio rispetto a quello di un'emodialisi con bicarbonato, l'unica voce di costo sfavorevole per i trattamenti "intensivi" viene sostanzialmente pareggiata.
L'ultimo nell'enunciazione abituale, ma, almeno secondo la visione anglosassone, il primo principio che regola la scelta bioetica, riguarda l'autonomia dei pazienti $(27,28,83-86)$. Apparentemente tutto è semplice: lasciamo che il paziente decida il proprio trattamento e, in questo ambito, il fatto che $i$ trattamenti in emodialisi domiciliare siano relegati a una nicchia più o meno consistente porta alcuni Autori a concludere aprioristicamente che si tratti di un trattamento di scarso interesse. Tuttavia, l'incremento dell'HHD nel mondo, che si registra attualmente dove questo trattamento viene sistematicamente offerto, sottolinea l'inconsistenza di questa posizione e, semmai, sposta il problema verso la necessità di offrire l'emodialisi domiciliare a tutti i pazienti, non solo in teoria, ma anche in pratica, creando dei Centri di riferimento accessibili, dove i pazienti possano ascoltare il parere di medici, infermieri e pazienti con esperienza specifica del trattamento in questione, superando le ovvie difficoltà di un'informazione da parte di chi non ha un'esperienza diretta. Ancora una volta, la letteratura è concorde in questo senso: i pazienti sono i migliori consiglieri di loro stessi, in assenza di controindicazioni cliniche. Se l'unica maniera di uscire dall'impasse di una non disponibilità-disinformazione passa attraverso un'offerta accessibile, il criterio di autonomia del paziente diventa il primo a sostenere un'implementazione sistematica di questo trattamento anche nel nostro Paese, peraltro in stretta analogia con quanto propugnato per la dialisi peritoneale, il cui riferimento dovrebbe innescare un circolo virtuoso di potenziamento delle cure domiciliari $(87,88)$ (Fig. 1).

\section{Dalla teoria alla pratica: una rapida disamina dei sistemi di implementazione della dialisi domiciliare nel mondo}

Se si segue la logica enunciata nei paragrafi precedenti (l'emodialisi domiciliare non è mai inferiore ed è, d'abitudine, superiore come risultati clinici e inferiore come costi rispetto all'emodialisi in Centro, non è in competizione, ma a complemento della dialisi peritoneale e, dove sistematicamente offerta, viene scelta da una percentuale non irrilevante dei pazienti), il problema chiave dell'emodialisi domiciliare si sposta verso l'identificazione del migliore (o dei migliori) modello di riferimento organizzativo.

Analogamente a quanto discusso rispetto ai costi, il modello di riferimento è strettamente legato al contesto socio-sanitario e si può modificare nel tempo; pertanto, quanto indicato in questo paragrafo non può che fornire indicazioni parziali e contestualizzate, che riassumono schemi di "azione" diversi, che possono, peraltro, essere modificati e adattati ulteriormente. In questo senso, l'analisi della dialisi si sposta dal singolo trattamento al sistema di cura, secondo un approccio chiamato "whole system research" che, sviluppato per le medicine cosiddette complementari o alternative, si presta bene all'analisi di terapie complesse, come la dialisi, in cui differenti alternative sono indissolubilmente legate (89-92). 
TABELLA I - RIMBORSI E RISPARMI CON UN PROGRAMMA DI EMODIALISI DOMICILIARE CON “TARGET" MINIMO DI 10 PAZIENTI; TALE COMPUTO, OTTENUTO DA UN PICCOLO CENTRO PIEMONTESE (SAN LUIGI, ORBASSANO), PERMETTE DI DIMOSTRARE COME SIA POSSIBILE INCREMENTARE ANCHE L'EMODIALISI DOMICILIARE, CON UNA STRATEGIA (IN QUESTO CASO PRIVILEGIANDO I PAZIENTI AD ALTO RISCHIO) CALATA NEL CONTESTO LOCALE (MODIFICATA DA G ITAL NEFROL 2012; 29 (2): 148-59)

Ia: Rimborsi

\begin{tabular}{|c|c|c|c|c|c|}
\hline & $\begin{array}{c}\text { Emodialisi ospedaliera } \\
\text { trisettimanale }\end{array}$ & $\begin{array}{c}\text { Emodialisi ospedaliera } \\
\text { quotidiana }\end{array}$ & $\begin{array}{c}\text { Emodiafiltrazione } \\
\text { ospedaliera trisettimanale }\end{array}$ & $\begin{array}{c}\text { Emodialisi domiciliare } \\
\text { trisettimanale }\end{array}$ & $\begin{array}{l}\text { Emodialisi domicilia- } \\
\text { re quotidiana }\end{array}$ \\
\hline Rimborso per seduta & $\begin{array}{c}189.9 \text { (165.3 per dialisi; } \\
20.7 \text { per piano } \\
\text { terapeutico; } 3.9 \text { per } \\
\text { medicazione FAV) }\end{array}$ & $\begin{array}{l}189.9(165.3 \text { per dialisi; } \\
\text { 20.7 per piano } \\
\text { terapeutico; } 3.9 \text { per medi- } \\
\text { cazione FAV) }\end{array}$ & $\begin{array}{l}\mathbf{2 8 2 . 8 5}(258.25 \text { per } \\
\text { dialisi, resto idem })\end{array}$ & $\begin{array}{l}118.8 \text { (seduta con piano } \\
\text { terapeutico eseguita men- } \\
\text { silmente in Centro, in } \\
\text { occasione dei controlli) }\end{array}$ & 118.8 (idem) \\
\hline Sedute/anno in Centro & 156 & 312 & 156 & 12 & 12 \\
\hline Sedute/anno domicilio & 0 & 0 & 0 & 144 & 300 \\
\hline Rimborso per anno & 29624.4 & 59248.8 & 44124.6 & 19386 & 37918.8 \\
\hline $\begin{array}{l}\text { Trasporto mensile } \\
\text { (1/mese per domiciliari) }\end{array}$ & $800-1000$ circa & $1500-2000$ circa & $800-1000$ circa & $80-100$ & $80-100$ \\
\hline Trasporto annuale & 10000 & 20000 & 10000 & 1000 & 1000 \\
\hline
\end{tabular}

Risparmio per la Regione, calcolato per 10 pazienti a domicilio vs in Centro

\begin{tabular}{lcccc}
\hline & $\begin{array}{c}\text { Emodialisi trisettimanale } \\
\text { Centro vs casa }\end{array}$ & \multicolumn{2}{c}{$\begin{array}{c}\text { Emodialisi quotidiana } \\
\text { Centro vs casa }\end{array}$} & $\begin{array}{c}\text { Emodiafiltrazione in Centro vs } \\
\text { emodialisi quotidiana casa }\end{array}$ \\
\hline Rimborso & 102384 & Rimborso & 213300 & Rimborso \\
Trasporti & 90000 & Trasporti & 190000 & Trasporti \\
Totale & $\mathbf{1 9 2 2 8 4}$ & Totale & $\mathbf{4 0 3 3 0 0}$ & $\begin{array}{c}\text { Totale } \\
(\mathbf{1 0} \text { pazienti/anno) }\end{array}$ \\
\hline
\end{tabular}

Ib: Costi diretti

Spese dirette ospedaliere, calcolato per 10 pazienti a domicilio e in Centro

\begin{tabular}{|c|c|c|c|c|c|}
\hline & $\begin{array}{c}\text { Emodialisi ospedaliera } \\
\text { trisettimanale }\end{array}$ & $\begin{array}{c}\text { Emodialisi } \\
\text { ospedaliera quotidiana }\end{array}$ & $\begin{array}{l}\text { Emodiafiltrazione ospe- } \\
\text { daliera trisettimanale }\end{array}$ & $\begin{array}{c}\text { Emodialisi domiciliare } \\
\text { trisettimanale }\end{array}$ & $\begin{array}{c}\text { Emodialisi domiciliare } \\
\text { quotidiana }\end{array}$ \\
\hline Costo kit di dialisi & 62400 & 124800 & 117000 & 117000 & 234000 \\
\hline $\begin{array}{l}\text { IP ( } 1 \text { IP/3-4 pazienti in Centro } \\
\text { - } 1 / 10 \text { pazienti a domicilio); } \\
\text { stipendio } 35100 / \text { anno }\end{array}$ & 100280 & $\begin{array}{c}100280 * \text { (calcolo a } \\
\text { paziente e non a dialisi) }\end{array}$ & 100280 & 35100 & 35100 \\
\hline $\begin{array}{l}\text { OSS ( } 1 \text { OSS per } 10 \text { pazienti in } \\
\text { Centro); stipendio } 25000 / \text { anno }\end{array}$ & 25000 & 25000 & 25000 & 0 & 0 \\
\hline Totale costi diretti & 187680 & 250080 & 242280 & 152100 & 269100 \\
\hline $\begin{array}{l}\text { Costi indiretti stimati ( } 20 \% \text { in } \\
\text { Centro, } 5 \% \text { dialisi domiciliare) }\end{array}$ & 37536 & 50016 & 48456 & 30420 & 53820 \\
\hline Totale costi diretti e indiretti & 225216 & 300096 & 290736 & 182520 & 322920 \\
\hline
\end{tabular}

Note:

a) "Kit dialisi": domiciliare: comprende la macchina per dialisi, l'assistenza e tutti i disposable, la consegna del materiale e il ritiro dei rifiuti a contatto con il sangue: costo medio piemontese: 75 Euro a seduta; ospedaliero: bicarbonato: 40 Euro (ns Centro); HDF (ns Centro) 75 Euro. Altre sedi riportano prezzi differenti, a seconda di quanto incluso nel "pacchetto dialitico" (Dr. Viganò: disposable e installazione: circa 35 Euro).

b) Il rimborso comprende: seduta dialitica, piano terapeutico (mensile a casa, quotidiano in Centro) e medicazione FAV (dati del tariffario Regionale Piemontese); il costo del kit di dialisi è stato calcolato sulla base dei bandi di gara più recenti disponibili in regione Piemonte per le tecniche impiegate.

c) Non sono contati i risparmi relativi a: giornate di lavoro perse, test ematochimici e ricoveri ospedalieri. Non sono contate le spese relative a personale medico, costo della struttura e costi di attivazione di nuovi Centri dialisi, necessari, salvo il potenziamento delle cure domiciliari, dato il costante incremento della popolazione in dialisi. Il computo non tiene conto dei costi dei farmaci. Nel computo totale non è contemplato il contributo economico di sostegno alla dialisi domiciliare regolamentato dai P.A.I.D.D. regionali, erogato sulla base del livello di autonomia e di intensità assistenziale del paziente. In Piemonte, l'incentivo può essere al massimo di 250 euro/mese per paziente e, in altre Regioni, come la Sicilia, fino a 350 euro. L'erogazione di questo contributo, che si può approssimare fino a 30000 euro/anno per 10 pazienti, non modifica in maniera sostanziale i calcoli riportati.

d) Non sono stati inseriti nella valutazione globale l'erogazione di 1200 euro per l'installazione dell'impianto al domicilio del paziente e 650 euro/anno di rimborso per le spese di luce e acqua. 


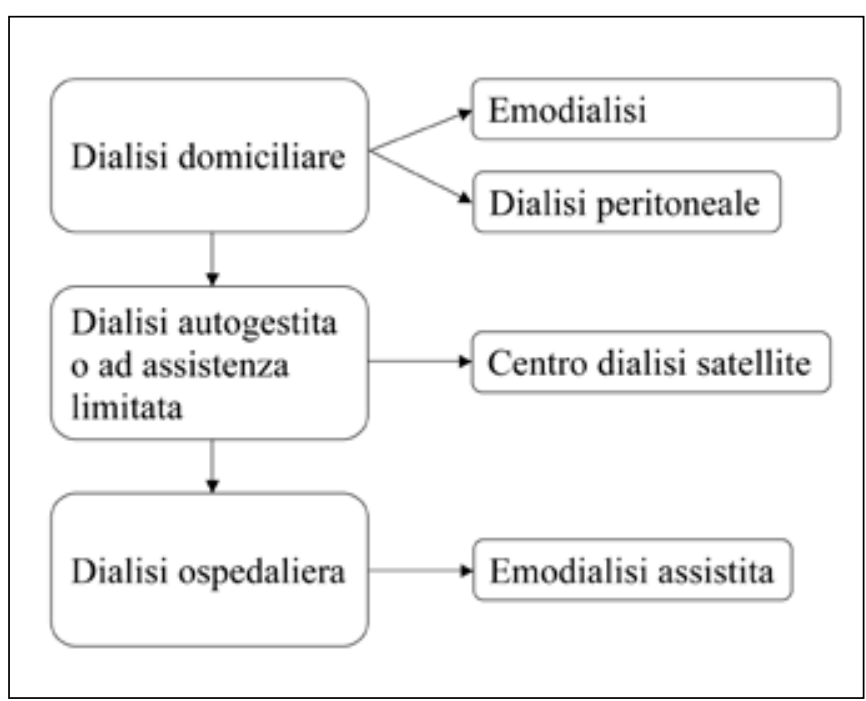

Fig. 1 - Il semplice paradigma decisionale "home dialysis first".

\section{Dialisi domiciliare, quotidiana breve "stile Perugia" (61, 65, 66, 69-71)}

Il gemellaggio tra emodialisi domiciliare e dialisi peritoneale, nell'economia organizzativa di un singolo Centro, può essere definito sotto l'egida dell'inventore dell'emodialisi domiciliare quotidiana breve, del primo apparecchio emodialitico portatile e del set a "Y". L'organizzazione della dialisi domiciliare "stile Perugia", al momento dei primi reali riconoscimenti internazionali dell'emodialisi quotidiana, rispondeva alle seguenti caratteristiche: unità medico-infermieristica dedicata a entrambi i trattamenti, stretto rapporto con il Centro dialisi, con disponibilità di dialisi quotidiana anche in Centro, trattamenti brevi (2-3 ore, 6-7 giorni alla settimana) e controlli adattati alle esigenze dei pazienti. Si tratta di un modello "monocentrico", che crea e mantiene una massa critica di pazienti combinando la dialisi peritoneale, spesso numericamente più consistente, con l'emodialisi domiciliare, privilegiando un approccio educazionale alla presa in carico da parte dei pazienti della propria malattia (quello che ora chiamiamo empowerment) rispetto alla differenziazione tecnologica tra i due tipi di dialisi. Lo stesso modello è tuttora in atto in alcune aree francesi, accanto, nelle grandi realtà urbane, come quella di Parigi, a una centralizzazione del trattamento in Unità di riferimento centralizzate (in questo caso, l'AURA).

\section{Dialisi notturna lunga "stile Toronto" $(17-19,21,22,29,44,45)$}

L'esperienza pionieristica di Buoncristiani e lo stretto legame tra emodialisi domiciliare e dialisi peritoneale, peraltro sulla scorta dell'insegnamento di un altro grande Maestro e inventore, il Professor Oreopoulos, ritornano nell'esperienza di Toronto, dove coesistono e trovano spazio due programmi analoghi di dialisi "notturna lunga". Si tratta di una sorta di contaminatio, nata alla fine del vecchio millennio, tra i due programmi non convenzionali di maggiore successo: la dialisi quotidiana alla Buoncristiani, in nuce domiciliare, e la dialisi notturna trisettimanale di Tassin $(93,94)$, che, anche se affiliata anche a un trattamento domiciliare, è essenzialmente una dialisi in Centro.

Il programma canadese nasce e si sviluppa con una certa rigidità di schema, anche figlia di una scelta di privilegiare una raccolta dati secondo criteri incontestabili, che permetta di dimostrare i vantaggi del trattamento "ad alta efficienza". Lo schema di base prevede 8 ore di dialisi per 6-7 notti alla settimana ed è squisitamente domiciliare. Il modello, ampiamente riprodotto in diverse sedi nordamericane, viene poi progressivamente a caratterizzarsi con una crescente flessibilità non solo con schemi dialitici (6-8 ore, 6-7 notti alla settimana, con occasionale opzione di "intervallo lungo" per permettere, per esempio, un fine settimana di libertà), ma anche di gestione, sia per l'inclusione nel programma di vari tipi di accesso vascolare e di entrambi i sistemi di venipuntura, button hole e ladder (95-97), sia per l'opzione di trattamento non assistito a domicilio, con o senza monitorizzazione a distanza. Anche se complesso da un punto di vista clinico e assistenziale, il programma ha l'indubbio vantaggio di dimostrare quelli che oggi sono i nuovi orizzonti della dialisi extracorporea: uno tra tutti, che in un certo senso li riassume, il successo delle gravidanze condotte in emodialisi notturna lunga, successo che rassicura sulla iatrogenicità di un trattamento che apporta una clearance equivalente sino a $40 \mathrm{~mL} / \mathrm{min}$, paragonabile (come, peraltro, anche se su piccola scala, lo sono i risultati) a quanto ottenibile con il trapianto renale $(19,21,98)$.

\section{Emodialisi domiciliare a schema multiplo: il modello australiano $(49,51,55)$}

L'emodialisi domiciliare è tuttora il trattamento di elezione di quasi il 10\% dei pazienti (e anche oltre, in alcune aree) in Australia e in Nuova Zelanda ed è in crescita netta in Canada. Senza cadere nella tentazione troppo facile di liquidare il successo dell'emodialisi domiciliare in queste sedi come legato solo alle grandi distanze di Paesi a bassa densità di popolazione, vi sono almeno due elementi di interesse generalizzabile in queste esperienze.

Il primo è la costituzione di Centri di riferimento ad ampio raggio, che seguono il training dei pazienti, controllano l'avvio a domicilio e coordinano, dove necessario e disponibile, l'appoggio con strutture vicine di riferimento in caso di necessità di rientro in Centro, per periodi di differente durata. Questo modello supera la necessità di offrire tutti i trattamenti in tutte le sedi e garantisce, per i trattamenti a minore prevalenza, un'esperienza specifica e una qualità elevata delle cure. Il secondo è la scelta di offrire l'intera rosa di trattamenti emodialitici, dalle dialisi notturne lunghe a quelle quotidiane brevi, ai ritmi trisettimanali standard. Questa flessibilità di scelta di trattamento sottolinea anche la preferenza, da parte della vasta maggioranza dei pazienti, di dialisi intensive, notturne lunghe e quotidiane brevi, a sancire, anche in questo contesto, il matrimonio felice tra dialisi quotidiana e domiciliare. 


\section{Dialisi breve assistita per pazienti critici:} il modello olandese $(43,99,100)$

Il modello sviluppato in Olanda si caratterizza per due peculiarità: la dialisi assistita a domicilio e la scelta di pazienti più critici per la dialisi a casa rispetto al Centro. In questo, il modello olandese rappresenta un rovesciamento della scelta abituale dei pazienti: non i soggetti autogestiti a basso rischio, ma i pazienti anziani per i quali la dialisi "dolce", a domicilio, senza necessità di trasporto, è da preferire alla dialisi trisettimanale in Centro. A riprova della sensibilità al contesto dell'argomento costi e della superficialità di un giudizio sulla dialisi quotidiana, sta il vantaggio economico che, almeno nei Paesi Bassi, viene attribuito al modello di dialisi domiciliare assistita, rispetto al Centro.

\section{La dialisi à la carte, il modello di Torino (4-6, 23-26, 57, 101)}

Fanalino di coda, la limitata esperienza di Torino si caratterizza per l'estrema flessibilità di trattamenti (da 1 a 7 dialisi, da 2 a 6 ore ciascuna), di training (da un mese a un anno, per affrontare non tanto il problema della conoscenza della macchina quanto l'accettazione del trattamento), di controlli (da 2 settimane a 2 mesi) e di legame con il Centro (sino a un'alternanza tra dialisi a domicilio e in Centro). Questo modello torinese, collaudato negli anni 1999-2005, è stato riproposto dal 2010 al 2012 in un'altra sede, con risultati analoghi dal punto di vista clinico ed economico (Tab. I). Anche se su piccola scala, questi dati suggeriscono che, anche nel nostro ambito, vi sia la possibilità di un'implementazione dell'emodialisi domiciliare in almeno un 1-3\% della popolazione, in linea con $\mathrm{i}$ migliori risultati ottenuti in altri Paesi europei.

\section{In conclusione}

L'emodialisi domiciliare non è una mummia fuggita dal museo, ma un trattamento flessibile, personalizzabile, economicamente vantaggioso e clinicamente promettente, almeno per un limitato numero di pazienti. Il crescente interesse che, in differenti parti del mondo, è nuovamente suscitato da questa metodica non può che portare a caldeggiare un reinserimento sistematico dell'offerta di emodialisi domiciliare anche nel panorama dialitico italiano.

\section{Riassunto}

La crisi economica globale, l'indicazione alla massima deospedalizzazione dei pazienti cronici e le innovazioni nel trattamento dell'uremia cronica sono alla base del rinnovato interesse per trattamenti dialitici "non convenzionali o intensivi", spesso od obbligatoriamente domiciliari. Un primo punto evidenziato da questa revisione narrativa del- la letteratura è il superamento dell'antagonismo storico tra emodialisi domiciliare (HHD) e dialisi peritoneale (PD), all'insegna del motto "home dialysis first": ascrivere il successo di una metodica domiciliare alla competizione con l'altra è riduttivo quanto il considerare che i pazienti ideali per l'HHD siano principalmente i soggetti che hanno dovuto interrompere la dialisi peritoneale.

Ciò detto, è possibile scomporre il problema della dialisi domiciliare non solo secondo criteri clinici, ma anche secondo i quattro principi etici di beneficio, non maleficio, giustizia e autonomia.

Se il beneficio non è facile da dimostrare, anche per la peculiare selezione dei pazienti, il non maleficio è evidente: in tutti gli studi analizzati, l'emodialisi domiciliare, sia essa standard o quotidiana o intensiva, non risulta mai significativamente inferiore ai trattamenti convenzionali. Il principio della giustizia, inteso in maniera un po' riduttiva come giustizia distributiva, può essere analizzato valutando i costi del trattamento, divisi tra costi diretti (disposable e macchine) e costo del personale medico e infermieristico; il vantaggio economico della riduzione del personale è ovvio, ma va anche ricordato che un sistema domiciliare necessita di una massa critica per essere favorevole dal punto di vista economico e che i costi "indiretti" (struttura ospedaliera in particolare) sono difficili da quantificare. Il quarto principio è l'autonomia dei pazienti: per questo sarebbe necessario offrire l'emodialisi domiciliare a tutti coloro che ne hanno le indicazioni, creando dei Centri di riferimento accessibili, dove i pazienti possano ascoltare il parere di medici, infermieri e pazienti con esperienza specifica.

Parole chiave: Dialisi domiciliare, Emodialisi, Dialisi pe ritoneale, Etica dei principi, Dialisi intensiva

Dichiarazione di conflitto di interessi: Gli Autori dichiarano di non avere conflitto di interessi.

Contributi economici agli Autori: Gli autori dichiarano di non aver ricevuto sponsorizzazioni economiche per la preparazione dell'articolo.

Indirizzo degli Autori:

Dr.ssa Giorgina Barbara Piccoli

Dipartimento di Scienze Cliniche e Biologiche

Università degli Studi di Torino

San Luigi Gonzaga, Regione Gonzole

10043 Orbassano (TO)

gbpiccoli@yahoo.it 


\section{Bibliografia}

1. Ikizler TA. Intensive hemodialysis: back to the beginning? J Am Soc Nephrol 2012; 23 (4): 573-5.

2. Lacson E Jr, Xu J, Suri RS, et al. Survival with three-times weekly in-center nocturnal versus conventional hemodialysis. J Am Soc Nephrol 2012; 23 (4): 687-95.

3. Nesrallah GE, Lindsay RM, Cuerden MS, et al. Intensive hemodialysis associates with improved survival compared with conventional hemodialysis. J Am Soc Nephrol 2012; 23 (4): 696-705.

4. Piccoli GB, Ferraresi M, Consiglio V, et al. Why home hemodialysis? A systematic "marketing" analysis. J Nephrol 2012; 25 (2): 159-69.

5. Piccoli GB. The never-ending search for the perfect dialysis. Should we move from the best treatment to the best system? Nephrol Dial Transplant 2011; 26: 1128-31.

6. Piccoli GB, Salomone M. Home hemodialysis: a system and not only a treatment. Kidney Int 2010; 78 (8): 819-20.

7. Blagg CR. Home haemodialysis. BMJ 2008; 336 (7634): 3-4.

8. Nosé Y. Home hemodialysis: a crazy idea in 1963: a memoir. ASAIO J 2000; 46: 13-7.

9. Merrill JP, Schupake E, Cameron E, Hampers CL. Hemodialysis in the home. JAMA 1964; 190: 468-70.

10. Curtis FK, Cole JJ, Tyler LL, Scribner BH. Hemodialysis in the home. Trans Am Soc Artif Intern Organs 1965; 11: 7-10.

11. Shaldon S. The true history of home haemodialysis. Nephrol Dial Transplant 2005; 20 (8): 1766-7.

12. Blagg CR, Daly SM, Rosenquist BJ, Jensen WM, Eschbach JW. The importance of patient training in home hemodialysis. Ann Intern Med 1970; 73: 841-7.

13. Delano BG. Whatever happened to home hemodialysis? J Dial 1977; 1: 465-7.

14. Woods JD, Port FK, Stannard D, Blagg CR, Held PJ. Comparison of mortality with home hemodialysis and center hemodialysis: a national study. Kidney Int 1996; 49: 1464-70.

15. Oberley ET, Schatell DR. Home hemodialysis: survival, quality of life, and rehabilitation. Adv Ren Replace Ther 1996; 3: 147-53.

16. Perl J, Chan CT. Home hemodialysis, Daily hemodialysis, and Nocturnal Hemodialysis: Core Curriculum 2009. Am J Kidney Dis 2009; 54: 1171-84.

17. Pierratos A, Nesrallah G. Current status of alternative hemodialysis regimens: an introduction. Semin Dial 2011; 24 (6): 605-6.

18. Rocco MV, Lockridge RS Jr, Beck GJ, et al. The effects of frequent nocturnal home hemodialysis: the Frequent Hemodialysis Network Nocturnal Trial. Kidney Int 2011; 80 (10): 1080-91.

19. Barua M, Hladunewich M, Keunen J, et al. Successful pregnancies on nocturnal home hemodialysis. Clin J Am Soc Nephrol 2008; 3 (2): 392-6.

20. Piccoli GB, Conijn A, Consiglio V, et al. Pregnancy in dialysis patients: is the evidence strong enough to lead us to change our counseling policy? Clin J Am Soc Nephrol 2010; 5 (1): 62-71.

21. Hladunewich M, Hercz AE, Keunen J, Chan C, Pierratos A. Pregnancy in end stage renal disease. Semin Dial 2011; 24 (6): 634-9.

22. Pauly RP, Gill JS, Rose CL, et al. Survival among nocturnal home haemodialysis patients compared to kidney transplant recipients. Nephrol Dial Transplant 2009; 24: 2915-9.

23. Piccoli GB, Bermond F, Mezza $\mathrm{E}$, et al. Home hemodialysis à la carte: a tailormade program (1998-2003). J Nephrol 2004; 17: 76-86.

24. Piccoli GB, Calderini M, Bechis F, et al. Daily dialysis Kt/V and flexible schedules: is it possible to control efficiency, when and how? Int J Artif Organs 2001; 24: 347-56.

25. Piccoli GB, Mezza E, Burdese M, et al. Dialysis choice in the context of an early referral policy: there is room for self care. $\mathrm{J}$ Nephrol 2005; 18 (3): 267-75.

26. Piccoli GB, Mezza E, Quaglia M, et al. Flexibility as an implementation strategy for a daily dialysis program. J Nephrol 2003; 16 (3): 365-72.

27. Moran J. The resurgence of home dialysis therapies. Adv Chronic Kidney Dis 2007; 14 (3): 284-9.

28. Agar JW, Mahadevan K, Knight R, Antonis ML, Somerville CA. 'Flexible' or 'lifestyle' dialysis: is this the way forward? Nephrology 2005; 10 (5): 525-9.

29. Raj DS, Charra B, Pierratos A, Work J. In search of ideal hemodialysis: is prolonged frequent dialysis the answer? Am J Kidney Dis 1999; 34 (4): 597-610.

30. Kooistra MP. Frequent prolonged home haemodialysis: three old concepts, one modern solution. Nephrol Dial Transplant 2003; 18 (1): 16-9.

31. Oreopoulos DG, Thodis E, Passadakis P, Vargemezis V. Home dialysis as a first option: a new paradigm. Int Urol Nephrol 2009; 41: 595-605.

32. Gonzalez-Perez JG, Vale L, Stearns SC, Wordsworth S. Hemodialysis for end-stage renal disease: a cost-effectiveness analysis of treatment-options. Int $\mathbf{J}$ Technol Assess Health Care 2005; 21: 32-9.

33. Zoccali C, Kramer A, Jager K. The databases: renal replacement therapy since 1989-the European Renal Association and European Dialysis and Transplant Association (ERA-EDTA). Clin J Am Soc Nephrol 2009; 4 (Suppl. 1): S18-22.

34. van de Luijtgaarden MW, Noordzij M, Stel VS, et al. Effects of comorbid and demographic factors on dialysis modality choice and related patient survival in Europe. Nephrol Dial Transplant 2011; 26 (9): 2940-7.

35. Castledine C, Gilg J, Rogers C, Ben-Shlomo Y, Caskey F. UK Renal Registry $13^{\text {th }}$ Annual Report (December 2010): Chapter 15: UK renal centre survey results 2010: RRT incidence and use of home dialysis modalities. Nephron Clin Pract 2011; 119 (Suppl. 2): c255-67.

36. Rhyn Lehmann P, Ambuehl M, Corleto D, Klaghofer R, Ambuehl PM. Epidemiologic trends in chronic renal replacement therapy over forty years: A swiss dialysis experience. BMC Nephrol 2012; 13 (1): 52.

37. Nadeau-Fredette AC, Bargman JM, Chan CT. Clinical outcome of home hemodialysis in patients with previous Peritoneal dialysis exposure: evaluation of the integrated home dialysis model. Perit Dial Int 2014.

38. Burkart J. Role of Peritoneal dialysis in the era of the resurgence of home hemodialysis. Hemodial Int 2008; 12 (Suppl. 1): S51-4.

39. Honkanen EO, Rauta VM. What happened in Finland to increase home hemodialysis? Hemodial Int 2008; 12 (Suppl. 1): S11-5. 
40. Malmström RK, Roine RP, Heikkilä A, et al. Cost analysis and health-related quality of life of home and self-care satellite haemodialysis. Nephrol Dial Transplant 2008; 23: 1990-6.

41. MacRae JM, Rose CL, Jaber BL, Gill JS. Utilization and outcome of 'out-of-center hemodialysis' in the United States: a contemporary analysis. Nephron Clin Pract 2010; 116: c53-9.

42. Johansen KL, Zhang R, Huang Y, et al. Survival and hospitalization among patients using nocturnal and short daily compared to conventional hemodialysis: a USRDS study. Kidney Int 2009; 76: 984-90.

43. Vanholder R, Davenport A, Hannedouche T, et al. Reimbursement of Dialysis: A Comparison of Seven Countries. J Am Soc Nephrol 2012; 23 (8): 1291-8.

44. Pauly RP, Maximova K, Coppens J, et al. Patient and technique survival among a Canadian multicenter nocturnal home hemodialysis cohort. Clin J Am Soc Nephrol 2010; 5: 1815-20.

45. McFarlane PA, Pierratos A, Redelmeier DA. Cost savings of home nocturnal versus conventional in-center hemodialysis. Kidney Int 2002; 62: 2216-22.

46. Kroeker A, Clark WF, Heidenheim AP, et al. An operating cost comparison between conventional and home quotidian hemodialysis. Am J Kidney Dis 2003; 42 (1 Suppl.): 49-55.

47. Lee H, Manns B, Taub K, et al. Cost analysis of ongoing care of patients with end-stage renal disease: the impact of dialysis modality and dialysis access. Am J Kidney Dis 2002; 40: 611-22.

48. Komenda P, Copland M, Makwana J, Djurdjev O, Sood MM, Levin A. The cost of starting and maintaining a large home hemodialysis program. Kidney Int 2010; 77: 1039-45.

49. Agar JW, Knight RJ, Simmonds RE, Boddington JM, Waldron CM, Somerville CA. Nocturnal haemodialysis: an Australian cost comparison with conventional satellite haemodialysis. Nephrology 2005; 10: 557-70.

50. Howard K, Salkeld G, White S, et al. The cost-effectiveness of increasing kidney transplantation and home-based dialysis. Nephrology 2009; 14: 123-32.

51. Agar JW, Hawley CM, Kerr PG. Home hemodialysis in Australia and New Zealand: how and why it has been successful. Semin Dial 2011; 24 (6): 658-63.

52. Kerr PG, Agar JW, Hawley CM. Alternate night nocturnal hemodialysis: the Australian experience. Semin Dial 2011; 24 (6): 664-7.

53. Marshall MR, Hawley CM, Kerr PG, et al. Home hemodialysis and mortality risk in Australian and New Zealand populations. Am J Kidney Dis 2011; 58 (5): 782-93.

54. Ludlow MJ, George CR, Hawley CM, et al. How Australian nephrologists view home dialysis: results of a national survey. Nephrology 2011; 16 (4): 446-52.

55. McClure DN. Home haemodialysis in Australia - is the wheel turning full circle? Med J Aust 2010; 193 (9): 559.

56. Pierratos A. Nocturnal hemodialysis: dialysis for the new millennium. CMAJ 1999; 161: 1137.

57. Piccoli GB, Bermond F, Mezza E, et al. Home hemodialysis. Revival of a superior dialysis treatment. Nephron 2002; 92: 324-32.

58. MacGregor MS, Agar JW, Blagg CR. Home haemodialysisinternational trends and variation. Nephrol Dial Transplant 2006; 21: 1934-45.
59. Masterson R. The advantages and disadvantages of home hemodialysis. Hemodial Int 2008; 12 (Suppl. 1): S16-20.

60. Nitsch D, Steenkamp R, Tomson CR, Roderick P, Ansell D, Macgregor MS. Outcomes in patients on home haemodialysis in England and Wales, 1997-2005: a comparative cohort analysis. Nephrol Dial Transplant 2011; 26: 1670-7.

61. Buoncristiani U, Quintaliani G, Cozzari M, Giombini L, Ragaiolo M. Daily dialysis: long-term clinical metabolic results. Kidney Int Suppl 1988; 24: S137-40.

62. Ting GO. The case for short daily hemodialysis, why sDHD will be the predominant modality for frequent dialysis. ASAIO J. 2001; 47: 443-5.

63. Pierratos A, McFarlane P, Chan CT. Quotidian dialysis-update 2005. Curr Opin Nephrol Hypertens 2005; 14: 119-24.

64. Basile C, Lomonte C. Nephrologists should strive for optimal haemodialysis: the case of 8-hour thrice-weekly in-centre haemodialysis. Nephrol Dial Transplant 2011; 26 (7): 2419-20.

65. Kjellstrand C, Buoncristiani U, Ting G, et al. Survival with short-daily hemodialysis: association of time, site, and dose of dialysis. Hemodial Int 2010; 14: 464-70.

66. Kjellstrand CM, Buoncristiani U, Ting G, et al. Short daily haemodialysis: survival in 415 patients treated for 1006 patient-years. Nephrol Dial Transplant 2008; 23: 3283-9.

67. Nesrallah GE, Suri RS, Carter ST, et al. The International Quotidian Dialysis Registry: annual report 2007. Hemodial Int 2007; 11: 271-7.

68. Blagg CR, Kjellstrand CM, Ting GO, Young BA. Comparison of survival between short-daily hemodialysis and conventional hemodialysis using the standardized mortality ratio. Hemodial Int 2006; 10: 371-4.

69. Buoncristiani U, Giombini L, Cozzari M, Carobi C, Quintaliani G, Brugnano R. Daily recycled bicarbonate dialysis with polyacrylonitrile. Trans Am Soc Artif Intern Organs 1983; 29: 669-72.

70. Buoncristiani U, Quintaliani G, Cozzari M, Giombini L, Ragaiolo M. Daily dialysis: long-term clinical metabolic results. Kidney Int Suppl 1988; 24: S137-40.

71. Buoncristiani U. Fifteen years of clinical experience with daily haemodialysis. Nephrol Dial Transplant 1998; 13 (Suppl. 6): 148-51.

72. Suri RS, Nesrallah GE, Mainra R, et al. Daily hemodialysis: a systematic review. Clin J Am Soc Nephrol 2006; 1 (1): 33-42.

73. Chertow GM, Levin NW, Beck GJ, et al. In-center hemodialysis six times per week versus three times per week. N Engl J Med 2010; 363 (24): 2287-300.

74. Twardowski ZJ, Misra M. "Daily" dialysis--lessons from a randomized, controlled trial. N Engl J Med 2010; 363 (24): 2363-4.

75. Finkelstein FO, Schiller B, Daoui R, et al. At-home short daily hemodialysis improves the long-term health-related quality of life. Kidney Int 2012; 82 (5): 561-9.

76. Weinhandl ED, Liu J, Gilbertson DT, Arneson TJ, Collins AJ. Survival in daily home hemodialysis and matched thriceweekly in-center hemodialysis patients. J Am Soc Nephrol 2012; 23 (5): 895-904.

77. Hine K. What is the outcome of applying principlism? Theor Med Bioeth 2011; 32 (6): 375-88.

78. Gordon JS. Global ethics and principlism. Kennedy Inst Ethics J 2011; 21 (3): 251-76. 
79. McCarthy J. Principlism or narrative ethics: must we choose between them? Med Humanit 2003; 29 (2): 65-71.

80. Pullman D. Ethics first aid: reframing the role of "principlism" in clinical ethics education and practice. J Clin Ethics 2005; 16 (3): 223-9.

81. Jindal KK. Commentary on the findings of the Canusa study. Perit Dial Int 1996; 16 (3): 246-7.

82. Quinn RR, Thorpe KE, Bargman JM. The higher risk of death on Peritoneal dialysis in the United States is not explained by background general population mortality: the Canusa study revisited. Clin Nephrol 2010; 73 (4): 286-93.

83. Lee A, Gudex C, Povlsen JV, Bonnevie B, Nielsen CP. Patients' views regarding choice of dialysis modality. Nephrol Dial Transplant 2008; 23 (12): 3953-9.

84. Wuerth DB, Finkelstein SH, Schwetz O, Carey H, Kliger AS, Finkelstein FO. Patients' descriptions of specific factors leading to modality selection of chronic Peritoneal dialysis or hemodialysis. Perit Dial Int 2002; 22 (2): 184-90.

85. Chanouzas D, Ng KP, Fallouh B, Baharani J. What influences patient choice of treatment modality at the pre-dialysis stage? Nephrol Dial Transplant 2012; 27 (4): 1542-7.

86. Goovaerts $\mathrm{T}$, Jadoul M, Goffin E. Influence of a pre-dialysis education programme (PDEP) on the mode of renal replacement therapy. Nephrol Dial Transplant 2005; 20 (9): 1842-7.

87. Gomes B, Calanzani N, Curiale V, McCrone P, Higginson IJ. Effectiveness and cost-effectiveness of home palliative care services for adults with advanced illness and their caregivers. Cochrane Database Syst Rev 2013; 6: CD007760.

88. Shepperd S, Iliffe S. Hospital at home versus in-patient hospital care. Cochrane Database Syst Rev 2001; (3): CD000356.

89. Ritenbaugh C, Verhoef M, Fleishman S, Boon H, Leis A. Whole systems research: a discipline for studying complementary and alternative medicine. Altern Ther Health Med 2003; 9 (4): 32-36.

90. Verhoef MJ, Mulkins A. The healing experience-how can we capture it? Explore 2012; 8 (4): 231-6.
91. Bell IR, Koithan M. Models for the study of whole systems. Integr Cancer Ther 2006; 5 (4): 293-307.

92. Elder C, Aickin M, Bell IR, et al. Methodological challenges in whole systems research. J Altern Complement Med 2006; 12 (9): 843-50.

93. Charra B, Calemard E, Ruffet M, et al. Survival as an index of adequacy of dialysis. Kidney Int 1992; 41: 1286-91.

94. Charra B, Terrat JC, Vanel T, et al. Long thrice weekly hemodialysis: the Tassin experience. Int J Artif Organs 2004; 27 (4): 265-83.

95. Parisotto MT, Schoder VU, Miriunis C, et al. Cannulation technique influences arteriovenous fistula and graft survival. Kidney Int 2014.

96. Chan MR, Shobande O, Vats H, et al. The Effect of Buttonhole Cannulation vs. Rope-ladder Technique on Hemodialysis Access Patency. Semin Dial 2014; 27 (2): 210-6.

97. Mustafa RA, Zimmerman D, Rioux JP, et al. Vascular access for intensive maintenance hemodialysis: a systematic review for a Canadian Society of Nephrology clinical practice guideline. Am J Kidney Dis 2013; 62 (1): 112-31.

98. Hladunewich MA, Hou S, Odutayo A, et al. Intensive Hemodialysis Associates with Improved Pregnancy Outcomes: A Canadian and United States Cohort Comparison. J Am Soc Nephrol 2014.

99. Cornelis T, Kotanko P, Goffin E, van der Sande FM, Kooman JP, Chan CT. Intensive hemodialysis in the (nursing) home: the bright side of geriatric ESRD care? Semin Dial 2012; 25 (6): 605-10.

100. Kooistra MP1, Vos J, Koomans HA, Vos PF. Daily home haemodialysis in The Netherlands: effects on metabolic control, haemodynamics, and quality of life. Nephrol Dial Transplant 1998; 13 (11): 2853-60.

101. Piccoli GB, Ferraresi M, Caputo F, et al. Home hemodialysis and Peritoneal dialysis compared. G Ital Nefrol 2012; 29 (2): 148-59. 